\title{
TESTING COSMOGONIC MODELS \\ WITH GRAVITATIONAL LENSING
}

\author{
JOACHIM WAMBSGANSS \\ Astrophysikalisches Institut Potsdam \\ An der Sternwarte 16 \\ 14482 Potsdam, Germany \\ AND \\ RENYUE CEN, JEREMIAH P. OSTRIKER, EDWIN L. TURNER \\ Princeton University Observatory \\ Peyton Hall \\ Princeton, NJ 08544 USA
}

\begin{abstract}
Gravitational lensing provides a strict test of cosmogonic models. We use fully non-linear numerical propagation of light rays through a model universe with inhomogeneities derived from a particular cosmogonic model, i.e. three-dimensional lensing simulations, to study its lensing properties. As a first example we present results for the standard CDM scenario. The lensing test for this model predicts that we should have seen far more widely split quasar images than have been found.
\end{abstract}

\section{Introduction}

Gravitational lensing directly measures fluctuations in the gravitational potential along lines of sight to distant objects. In contrast, the conventional tools for comparing theories with observations rely on either galaxy density or velocity information, both of which unavoidably suffer from the uncertainties with regard to density or velocity bias of galaxies over the underlying mass distribution, hampering our attempts to understand the more "fundamental" questions concerning the mass evolution and distribution. Thus, gravitational lensing provides a powerful independent test of cosmogonic models (Narayan \& White 1988). Each model for the de- 
velopment of cosmogonic structure (e.g. the hot dark matter or cold dark matter [CDM] scenario) has at least one free parameter, the amplitude of the density power spectrum. But now in the light of COBE observations (Smoot et al. 1992) that parameter is fixed by the $( \pm 15 \%)$ determination on the $5^{\circ}-10^{\circ}$ scale in the linear regime. With its amplitude fixed, a secure determination of the potential fluctuation on any scale provides a test; any single conflict between the theory and reality can falsify the former. The most leverage is obtained for tests made on scales as far as possible from the COBE measurements, since all models have an assumed power spectrum that passes through the COBE normalization point at the very large comoving scales $(\lambda \approx 1000 \mathrm{Mpc})$ fixed by that measurement. Since the slope of the power spectrum is a primary model dependent feature, the maximum variations amongst models occur at the smallest scales. Thus one looks for tests at scales as small as possible, but they should not be so small as to be greatly influenced by the difficulty in modeling the physics of the gaseous, baryonic components $(\leq 10 \mathrm{kpc})$. Thus critical tests are best made on scales $0.01 \mathrm{Mpc}<r<1 \mathrm{Mpc}$. Here we show how to use gravitational lensing from matter distributions on these scales to test cosmogonic models, with the standard CDM scenario as our first example. More detailed descriptions of the results and the method can be found in Wambsganss et al. (1995a,b).

\section{Cosmological Model}

The cosmogonic model tested here is the "standard" CDM scenario with $\Omega=1, \lambda=0$ and $H_{0}=100 h=50 \mathrm{~km} \mathrm{~s}^{-1} \mathrm{Mpc}^{-1}$. Normalization, taken from the COBE first year results (Smoot et al. 1992), corresponds to $\sigma_{8}=1.05$. In order to allow for the existence of very large-scale waves, we first ran an $L=400 h^{-1} \mathrm{Mpc}$ size box with $500^{3}$ cells and $250^{3}$ particles. In order to have detailed small scale information we reran a total of 10 independent simulations with $L=5 h^{-1} \mathrm{Mpc}$, with $500^{3}$ cells and $250^{3}$ particles. Knowing the distribution of overdensities on the $5 h^{-1} \mathrm{Mpc}$ scale from the large simulation, we statistically convolve the small and large scale runs to produce simulated sheets or screens of matter spaced $5 h^{-1} \mathrm{Mpc}$ apart between the observer at $z=0$ and a putative galaxy or quasar in the source plane at $z=z_{S}$. A large number of independent runs (ten were simulated) is required so that identical structures do not repeat along a line of sight. Details and tests of the convolution method are presented in Wambsganss et al. (1995b). It is statistically reliable for describing structures in the range $30 \mathrm{kpc}<\Delta L h<1.2 \mathrm{Mpc}$, which corresponds roughly to splitting angles $5 "<\theta<200$ ". On these scales we expect that dark matter dominates over baryons so that a dark matter only simulation is approximately valid. 


\section{Lensing Method}

We "fill" the universe densely with adjacent matter cubes, which are obtained from cosmological simulations as described above. Inside each cube, we project the matter onto the mid plane perpendicular to the line of sight. Then, we follow light rays through all lens planes. We speed up the calculation of deflection angles by use of an hierarchical tree code. Typically we use a few hundred (grouped) screens for each "line of sight". In a source plane at a given redshift, we then determine various lensing properties of this particular line of sight. The magnification in the source plane is simply given by the density of rays, relative to the unlensed case (see Figure 1a for one particular line of sight). Similarly, the magnification in the image plane is obtained by the differential area within a bundle of rays as compared to what it would have been had the propagation been through a universe with smoothly distributed matter (Figure 1b). Naturally we allow for crossing of ray bundles, i.e. multiple imaging. We can then use any distribution of sources (positions, sizes, shapes, redshifts) in order to determine their properties after being lensed by this three dimensional matter distribution. As an example, in Figure 1c we show the "images" of a regular grid of circular sources at $z_{S}=3.0$. Deformation and change of source sizes are quite obvious in the regions that are highly magnified. In Figure 1d the "average" shear in areas of $(20 \mathrm{arcsec})^{2}$ is shown for this particular line of sight.

\section{Results}

For 100 different realizations, we have computed the distribution of magnifications for single and multiply-imaged point sources as a function of $z_{S}$, as well as multiplicity of images, distribution of angular splittings, rms shear and other properties. In addition, for extended sources, we have computed the expected shape distortions, frequency and properties of the giant arcs that would be seen, when the sources are lensed by intervening clusters. Figure 2a shows the probability of a splitting with separation of images greater than 5" and magnitude difference less than $1.5 \mathrm{mag}$ as a function of source redshift. In fact, amplification bias will increase the probabilities over those shown in Figure 2a by a significant amount. Splittings larger than 5" should be common (when several thousand quasars have been examined), if this cosmogonic model were correct. Probably the single most revealing statistic is the distribution of image separations expected for multiple sources as shown in Figure $2 \mathrm{~b}$ for $z_{S}=1,2$ and 3 . Notice that very large splittings should be the rule. Also revealing is the distribution of expected lens redshifts as shown in Figure 2c. The lenses themselves should be close enough to be seen in almost all cases. On this issue the recent observation of a lens candidate for the double quasar QSO2345+007 (Fis- 


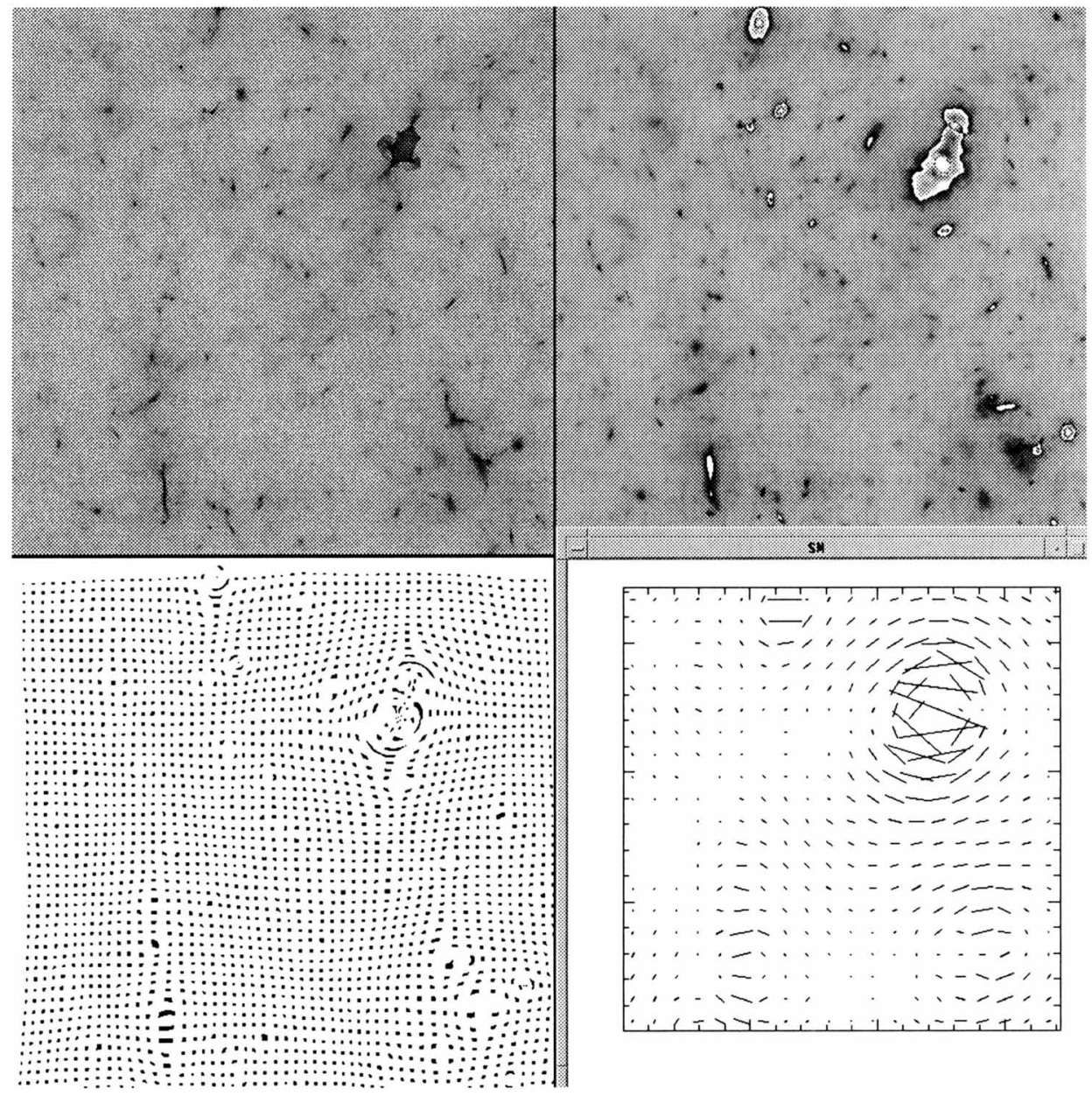

Figure 1. a) (top left) Example of the magnification due to the gravitational lens action of a CDM, $\Omega=1$ universe for a source plane at $z_{S}=3.0$. The size of the field is about $(5.7 \text { arcmin })^{2}$. The gray scale indicates magnification, dark means high magnification. The sharp dark boundaries (caustics) indicate regions of multiple imaging. b) (top right) Magnification in the image plane. Again, dark indicates high magnification. The white regions just inside very dark regions indicate areas with formally negative magnification, i.e. regions that contain multiple images. The boundaries between the black and the white regions are the critical lines. c) (bottom left) "Image" of a regular grid of circular sources at a redshift of $z_{S}=3.0$ for this particular line of sight. One clearly sees the effect of the lensing: variations in size indicate magnification as function of position. One can see arclets, arcs, and even multiple images (i.e., a row or column breaks up into more than one track). Interpreted as images of point sources, the relative sizes reflect the intensity ratio. d) (bottom right) Shear distribution for this line of sight: shear is averaged in square regions of about 20 arcsec at a side. The lines indicate the direction and the relative strength of the shear. 

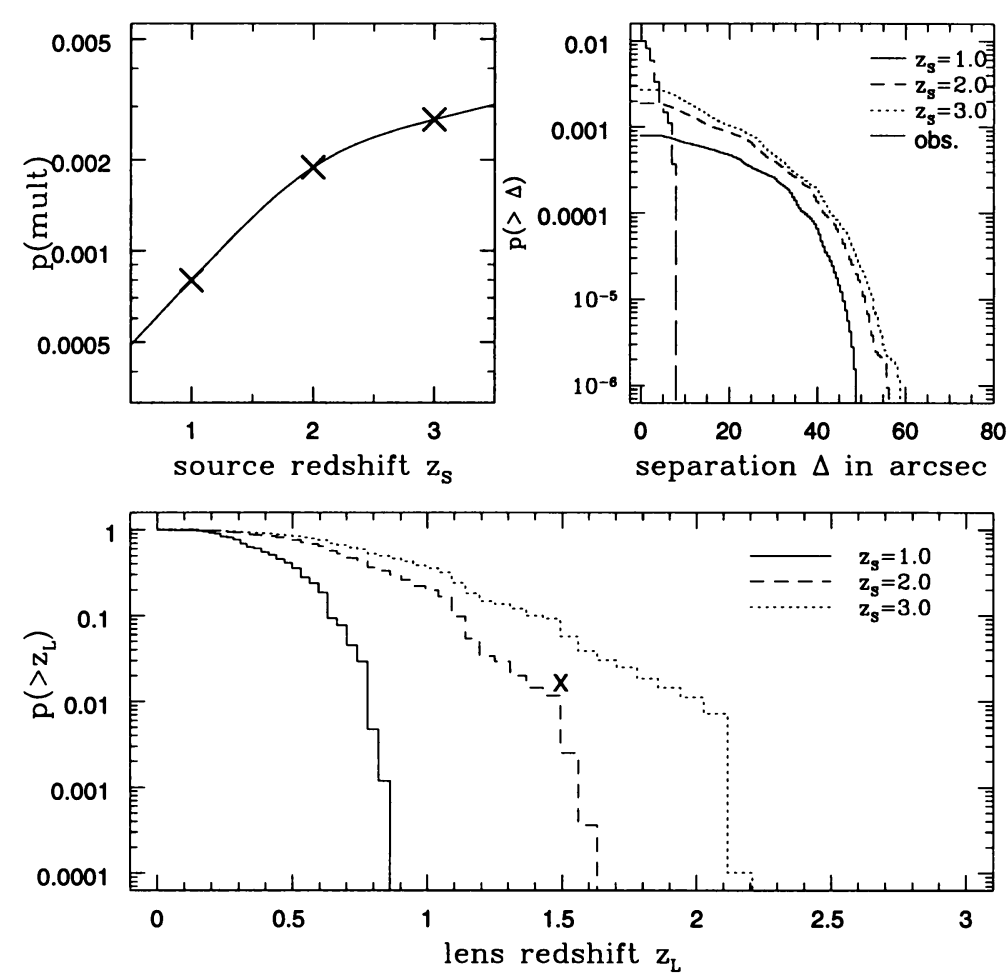

Figure 2. a) (top left) Probability of multiple imaging as a function of source redshift. Only cases with separation of images greater than 5 " and less than $1.5 \mathrm{mag}$ difference are considered. b) (top left) Multiple-lensing probability distribution as a function of image separations for sources at $z_{S}=1,2$ and 3 . Also shown as long dashed curve is the observed distribution (cf. Surdej \& Soucail 1993). c) (bottom) Integrated lensing probability distribution as a function of expected lens redshift. The " $X$ " indicates the recent observation (Fischer et al. 1994) of a lens candidate for the double quasar QSO2345+007.

cher et al. 1994) is extremely relevant. The separation of the two images is 7.06 arcsec, the quasar redshift is $z_{S}=2.15$ and the putative lens is at $z_{L}=1.49$. We see from Figure $2 \mathrm{c}$ that, although 7 arcsec separation can be produced in the CDM model, the probability that the lens is as far away as $z=1.49$ is very small ( $2 \%)$ due to the relatively late formation of structure in this model. In open models structure formation occurs earlier.

It appears that all three of these results (shown in Figures 2a,b,c) are seriously in conflict with the existing observations. In particular, we find that the standard CDM model predicts that 0.0007 of all lines of sight to $z_{S}=1,0.0014$ of all lines of sight to $z_{S}=2$ and 0.0020 of those to $z_{S}=3$ will be multiply-imaged with angular splittings $\geq 10$ " and amplifi- 
cation ratios of less than 1.5 magnitudes. Various surveys and occasional serendipitous discoveries have revealed 27 confirmed or possible multiplyimaged QSO's according to a recent compilation (Surdej \& Soucail 1993; an updated version of this statistics can be found in this volume). Detailed analysis of these surveys yields a lensing rate in the vicinity of a few tenths to one percent, consistent with the CDM predictions quoted above making allowance for plausible magnification biases. However, as shown in Figure $2 \mathrm{~b}$, all observed QSO lens systems have image splittings of less than 10 ", and the large majority, less than 5". This sharply contradicts and thus falsifies the model. Since the large splitting, modest brightness ratio systems predicted by the model would be typically much easier to detect and recognize than those 27 which have actually been found, no escape by appeal to observational selection seems possible. A similar conclusion for this cosmogonic model has been found by Kochanek (1995) in a semi-analytic study of the lensing properties of various cosmological models.

This failing of the model is not presented as an entirely new result, but only as a new and more robust manifestation of a previously recognized problem, namely the excessively deep potential wells produced by the dark matter component in COBE normalized standard CDM. These potential wells lead both to excess galaxy pair-wise velocity dispersions and to the predicted excessive rate of large splitting lensing events. The virtue of the lensing test is that it is independent of other tests and is not subject to the same caveats concerning "bias" of galaxies with respect to dark matter. We are in the process of testing other models ( e.g. low $\Omega$; or $\Lambda$ dominated). The directness of gravitational lensing as a test for the growth of inhomogeneities, coupled with the rapidly increasing power of computers and numerical algorithms, makes one optimistic that calculations of the type reported on here should become a major tool for testing and discriminating among competing cosmological scenarios.

\section{References}

Fischer, P., Tyson, J.A., Bernstein, G.M., \& Guhathakurta, P., 1994, ApJL, 431, L71

Kochanek, C.S., 1995, ApJ, in press

Narayan, R. \& White, S.D.M., 1988, MNRAS, 231, 97p

Smoot, G.F., et al., 1992, ApJL, 396, L1

Surdej, J., \& Soucail, G., 1993, in Gravitational Lenses in the Universe, eds. J. Sudej, D.

Fraipont-Caro, E. Gosset, S. Refsdal, \& M. Remy, (Liège: Université de Liège) 205

Wambsganss, J., Cen, R., Ostriker, J. P., \& Turner, E. L., 1995a, Science, 268, 274

Wambsganss, J., Cen, R., Ostriker, J. P., 1995b, ApJ, submitted 